\title{
Factoring Zero-dimensional Ideals of Linear Partial Differential Operators
}

\author{
Ziming $\mathrm{Li}^{*}$ \\ Symbolic Computation Group \\ Dept. of Computer Science \\ Univ. of Waterloo, Waterloo \\ Ontario, Canada, N2L 3G1 \\ z6li@scg.math. uwaterloo.ca
}

\author{
Fritz Schwarz \\ FhG, Institut SCAI \\ 53754 Sankt Augustin \\ Germany \\ fritz.schwarz@gmd.de
}

\author{
Serguei P. Tsarev ${ }^{\dagger}$ \\ Dept. of Math, Krasnoyarsk \\ State Pedagogical Univ. \\ Lebedevoi 89, 660049 \\ Krasnoyarsk, Russia \\ tsarev@edk. krasnoyarsk.su
}

\begin{abstract}
We present an algorithm for factoring a zero-dimensional left ideal in the ring $\overline{\mathbf{Q}}(x, y)\left[\partial_{x}, \partial_{y}\right]$, i.e. factoring a linear homogeneous partial differential system whose coefficients belong to $\overline{\mathbf{Q}}(x, y)$, and whose solution space is finite-dimensional over $\overline{\mathbf{Q}}$. The algorithm computes all the zero-dimensional left ideals containing the given ideal. It generalizes the BekeSchlesinger algorithm for factoring linear ordinary differential operators, and uses an algorithm for finding hyperexponential solutions of such ideals.
\end{abstract}

\section{INTRODUCTION}

For various reasons linear differential equations have been of particular importance in the history of mathematics. First of all, the problems connected with them are much easier than those for nonlinear equations. Second, many nonlinear problems may be linearized in some way such that the results of the former theory may be applied to them. This is especially true for Lie's symmetry analysis of differential equations which reduces the problem of solving nonlinear ordinary differential equations (ode's) with a sufficiently large number of symmetries to the study of certain systems of linear partial differential equations (pde's). The problem of finding conservation laws for nonlinear pde's also leads to systems of linear pde's.

It has been possible to generalize many concepts from commutative algebra suitably such that they may be applied to linear ode's, e.g. the greatest common divisor and least com-

\footnotetext{
*Partially supported by Natural Sciences and Engineering Research Council of Canada Grant No. CRD215442-98 and by a National Key Basic Research Project of China (No. G1998030600).

†Partially supported by INTAS Grant 99-01782 and Russian Ministry of Education Grant E00-1.0-57.
}

Permission to make digital or hard copies of all or part of this work for personal or classroom use is granted without fee provided that copies are not made or distributed for profit or commercial advantage, and that copies bear this notice and the full citation on the first page. To copy otherwise, to republish, to post on servers or to redistribute to lists, requires prior specific permission and/or a fee.

ISSAC 2002, July 7-10, 2002, Lille, France

(C) 2000 ACM 1-58113-484-3/ 02/0007

$\$ 5.00$ mon multiple, the concept of reducibility and factorization which finally led to the theory of Picard and Vessiot and differential Galois theory. This is true to a much less extent for systems of linear pde's. In order to obtain manageable problems, we have to specialize them further. The constraint that the general solution depends on a finite number of constants, i.e. that it may be represented as a linear combination with constant coefficients of a finite number of special solutions which form a fundamental system, turns out to be appropriate. It allows us to generalize many concepts from the theory of linear ode's in an almost straightforward manner to these pde's. Furthermore, they arise from different research areas such as: symmetry analysis, holonomic differential systems [9] and representations of functions in terms of pde's and initial values [3].

It is the purpose of this paper to describe a generalization of the Beke-Schlesinger factorization algorithm $[1,10]$ to systems of linear homogeneous pde's in one dependent and two independent variables with a finite-dimensional solution space. The base field consists of the rational functions in the independent variables with algebraic number coefficients. Such an algorithm may have interesting applications in the above-mentioned areas. In principle, most problems related to such systems of pde's reduce, as shown by S. Lie, to corresponding problems for linear ode's. However, such "reduction" may be nontrivial and usually leads to solving or factoring linear ode's with parameters. This makes many known algorithms fail. The algorithm presented in this paper avoids such complications.

In this paper the following notation will be used: $\overline{\mathbf{Q}}$ stands for the algebraic closure of the field of rational numbers, $\mathbf{K}$ for the differential field $\overline{\mathbf{Q}}(x, y)$ with usual derivation operators $\partial_{x}$ and $\partial_{y}$, and $\mathbf{K}\left[\partial_{x}, \partial_{y}\right]$ for the ring of linear partial differential operators generated by $\partial_{x}$ and $\partial_{y}$ over $\mathbf{K}$. By an ideal in $\mathbf{K}\left[\partial_{x}, \partial_{y}\right]$ we mean a left ideal in $\mathbf{K}\left[\partial_{x}, \partial_{y}\right]$. A system of linear homogeneous pde's in $\partial_{x}$ and $\partial_{y}$ over $\mathbf{K}$ can be naturally identified with an ideal in $\mathbf{K}\left[\partial_{x}, \partial_{y}\right]$ (see, e.g. $[9, \S 1.1])$. A solution of a system is understood as an element of a universal differential field extension $\mathbf{E}$ of $\mathbf{K}$, which is annihilated by the operators in the corresponding ideal of $\mathbf{K}\left[\partial_{x}, \partial_{y}\right]$. A system has a finite-dimensional solution space over $\overline{\mathbf{Q}}$ if and only if the corresponding ideal is zero-dimensional (abbreviated as: 0-dim). This point of 
view enables us to make statements concisely. In this algebraic setting, our factorization algorithm computes all ideals (in $\mathbf{K}\left[\partial_{x}, \partial_{y}\right]$ ) containing a given 0-dim ideal. Factoring a 0 -dim ideal in $\mathbf{K}\left[\partial_{x}, \partial_{y}\right]$ is more involved than factoring a linear ode. For instance, the notions of factors and quotients need to be carefully examined; the leading derivatives of a factor are not as obvious as in the ode case; the notion of Wronskians will be extended; and the normal form of a factor has to be considered. Most of the results stated in this paper hold for several variables. We confine ourselves to the case of two variables, because a generalization of the algorithm in [7] for several variables is still on the way.

The paper is based on several known results. The theory of linear differential ideals [6] supplies useful conclusions about dimension and linear dependence over a constant field. The notion and computation of differential Gröbner bases in $\mathbf{K}\left[\partial_{x}, \partial_{y}\right]$ (see $[5,3,9]$ ) make sure that the system to be factored and the factors to be sought are of required dimensions. The algorithm in [7] enables us to compute firstorder factors. The idea of associated equations $[1,10,11$, 2 ] inspires us to reduce our factorization problem to that of finding first-order factors. The factorization problem in this paper has been considered by Tsarev [14], in which notions of quotients and associated equations are introduced. But the results about algorithms therein are quite sketchy and contain a mistake (see the end of Section 4 of this paper). We shall describe these notions and algorithms precisely, and provide proofs and examples.

The paper is organized as follows. Section 2 specifies the notation and states our factorization problem. Section 3 discusses quotient systems. Section 4 presents some simple and useful facts about factorization. Sections 5 and 6 generalize the notions of Wronskians and associated systems, respectively. Section 7 describes ideas about factorization. Section 8 presents a factorization algorithm. Concluding remarks are given in Section 9.

\section{PRELIMINARIES}

We denote by $\Theta$ the commutative monoid generated by $\partial_{x}$ and $\partial_{y}$. An element of $\Theta$ is called a derivative. For a subset $L$ of $\mathbf{K}\left[\partial_{x}, \partial_{y}\right]$, $\operatorname{sol}(L)$ stands for the solution space of $L$, which is contained in $\mathbf{E}$ and is a vector space over $\overline{\mathbf{Q}}$. The ideal generated by $L$ is denoted by $(L)$. Two ideals of $\mathbf{K}\left[\partial_{x}, \partial_{y}\right]$ are equal if and only if their solution spaces are equal [6, page, 151]. An ideal $I$ of $\mathbf{K}\left[\partial_{x}, \partial_{y}\right]$ is said to be 0 -dim if the left linear space $\mathbf{K}\left[\partial_{x}, \partial_{y}\right] / I$ is of finite dimension over $\mathbf{K}$. It follows from [6, Chap. IV, $\S 5]$ that $\operatorname{dim}_{\mathbf{K}}\left(\mathbf{K}\left[\partial_{x}, \partial_{y}\right] / I\right)=\operatorname{dim}_{\overline{\mathbf{Q}}} \operatorname{sol}(I)$ if the ideal $I$ is 0 -dim.

For convenience, we fix a term order

$$
1<\partial_{x}<\partial_{y}<\partial_{x}^{2}<\partial_{x} \partial_{y}<\partial_{y}^{2}<\cdots
$$

on $\mathbf{K}\left[\partial_{x}, \partial_{y}\right]$ throughout the paper. The reader may easily find that all conclusions in this paper are valid for any term order. A differential Gröbner basis in $\mathbf{K}\left[\partial_{x}, \partial_{y}\right]$ will be simply called a Gröbner basis. By "given an ideal", we mean to be given its reduced Gröbner basis. In this paper, Gröbner bases may be replaced by (linear) coherent autoreduced sets $[8,6]$, or by Janet bases $[4,12,13]$, both of which appeared earlier in the literature than Gröbner bases. We choose Gröbner bases because they are well-known and their computer algebra implementations are widely available. For brevity, all Gröbner bases are assumed to be reduced. It seems improper to factor an arbitrary system, for such a system may possibly be inconsistent. For a Gröbner basis $L$, we denote by $\operatorname{lder}(L)$ the set of leading derivatives of $L$, and by $\operatorname{pder}(L)$ the set of parametric derivatives of $L$. Recall that a leading derivative of $L$ is the highest derivative in an element of $L$, and a parametric derivative of $L$ is a derivative not divisible by any element of $\operatorname{lder}(L)$. By $[6$, Chap. IV,$\S 5]$ or $[9, \S 1.4], \operatorname{dim}_{\overline{\mathbf{Q}}} \operatorname{sol}(L)=|\operatorname{pder}(L)|$ if $(L)$ is 0 -dim. Both $|\operatorname{lder}(L)|$ and $|\operatorname{pder}(L)|$ can be obtained from $L$.

This paper provides an algorithm for solving

Problem F Given a Gröbner basis $L$ with $\operatorname{dim}_{\overline{\mathbf{Q}}} \operatorname{sol}(L)=d$ and an integer $n$ with $1 \leq n<d$, compute all Gröbner bases $F$ in $\mathbf{K}\left[\partial_{x}, \partial_{y}\right]$ such that

$$
\begin{aligned}
& \text { 1. } \operatorname{dim}_{\overline{\mathbf{Q}}} \operatorname{sol}(F)=n \text {, or, equivalently, }|\operatorname{pder}(F)|=n \text {; } \\
& \text { 2. } \operatorname{sol}(F) \subset \operatorname{sol}(L) \text {, or, equivalently, }(L) \subset(F) \text {. }
\end{aligned}
$$

We call $F$ an $n$ th-order factor of $L$ over $\mathbf{K}$. By "computing a factor of $L$ ", we mean to compute the reduced Gröbner basis for the factor.

EXAMPLE 1. Let $L$ be the Gröbner basis:

$$
\begin{gathered}
\left\{L_{1}=\partial_{x}^{2}+\frac{y^{2}}{4 x} \partial_{y}+\frac{2-x y}{4 x} \partial_{x}-\frac{y}{4 x},\right. \\
L_{2}=\partial_{x} \partial_{y}+\frac{y}{4} \partial_{y}-\frac{2+x y}{4 y} \partial_{x}-\frac{1}{4}, \\
\left.L_{3}=\partial_{y}^{2}+\frac{x}{4} \partial_{y}+\frac{2 x-x^{2} y}{4 y^{2}} \partial_{x}-\frac{x}{4 y}\right\} .
\end{gathered}
$$

We find $\operatorname{lder}(L)=\left\{\partial_{x}^{2}, \partial_{x} \partial_{y}, \partial_{y}^{2}\right\}$ and $\operatorname{pder}(L)=\left\{\partial_{x}, \partial_{y}, 1\right\}$. Hence, $\operatorname{sol}(L)$ is of dimension 3 over $\overline{\mathbf{Q}}$. The Gröbner basis

$$
F=\left\{\partial_{x}^{2}+\frac{1}{2 x} \partial_{x}-\frac{y}{4 x}, \partial_{y}-\frac{x}{y} \partial_{x}\right\}
$$

is a second-order factor of $L$, since $L_{1}, L_{2}$ and $L_{3}$ can be reduced to zero by $F$. Notice that $(\operatorname{lder}(L) \cap \operatorname{lder}(F))$ is nonempty, which never happens in the ode case. One may observe that (1) also has a first-order factor $\left\{\partial_{y}-\frac{1}{y}, \partial_{x}\right\}$.

REMARK 2. As pointed out in the proof of Theorem 2 in [14], the solution spaces of systems in $\mathbf{K}\left[\partial_{x}, \partial_{y}\right]$ contained in $\operatorname{sol}(L)$ form a module lattice $\mathbf{V}$ in which the partial ordering is inclusion. For $V_{1}, V_{2} \in \mathbf{V}$, both $\left(V_{1} \cap V_{2}\right)$ and $\left(V_{1}+V_{2}\right)$ are also in $\mathbf{V}$. Indeed, they correspond to the sum (gcd) and intersection $(\mathrm{lcm})$ of respective defining ideals. Hence, for two maximal chains of proper factors $F_{1}, \ldots, F_{k}$ and $G_{1}$, $\ldots, G_{m}$ with

$$
\{0\} \subset \operatorname{sol}\left(F_{1}\right) \subset \cdots \subset \operatorname{sol}\left(F_{k}\right) \subset \operatorname{sol}(L)
$$

and

$$
\{0\} \subset \operatorname{sol}\left(G_{1}\right) \subset \cdots \subset \operatorname{sol}\left(G_{m}\right) \subset \operatorname{sol}(L),
$$

we have $m=k$ and the quotient spaces $\left(\operatorname{sol}\left(F_{i}\right) / \operatorname{sol}\left(F_{i-1}\right)\right)$ and $\left(\operatorname{sol}\left(G_{j}\right) / \operatorname{sol}\left(G_{j-1}\right)\right)$ have the same dimension pairwise after a rearrangement of indices. 
In the rest of this paper, $L \subset \mathbf{K}\left[\partial_{x}, \partial_{y}\right]$ is assumed to be a Gröbner basis with $\operatorname{dim}_{\overline{\mathbf{Q}}} \operatorname{sol}(L)=d>0$.

To save space, we use exterior algebra notation to denote determinants. For $\theta_{1}, \ldots, \theta_{n} \in \Theta$, the exterior product

$$
\lambda=\theta_{1} \wedge \theta_{2} \wedge \cdots \wedge \theta_{n}
$$

is understood as a multi-linear mapping from $\mathbf{E}^{n}$ to $\mathbf{E}$ :

$$
\lambda(\vec{z})=\left|\begin{array}{cccc}
\theta_{1} z_{1} & \theta_{1} z_{2} & \cdots & \theta_{1} z_{n} \\
\theta_{2} z_{1} & \theta_{2} z_{2} & \cdots & \theta_{2} z_{n} \\
\vdots & \vdots & \cdots & \vdots \\
\theta_{n} z_{1} & \theta_{n} z_{2} & \cdots & \theta_{n} z_{n}
\end{array}\right|
$$

for $\vec{z}=\left(z_{1}, \ldots, z_{n}\right) \in \mathbf{E}^{n}$. Besides being multi-linear and anti-symmetric, $\lambda$ also has the property that, for all $a \in \mathbf{K}$,

$$
\partial_{x}(a \lambda)=\left(\partial_{x} a\right) \lambda+a \sum_{j=1}^{n}\left(\theta_{1} \wedge \cdots \wedge \partial_{x} \theta_{j} \wedge \cdots \wedge \theta_{n}\right) .
$$

In this way we may regard any $\mathbf{K}$-linear combination of $n$ fold exterior products formed by elements of $\Theta$ as a multilinear function from $\mathbf{E}^{n}$ to $\mathbf{E}$. Clearly, a derivation operator can be applied to such a combination. For a subset $S$ of $\Theta$, we denote by $\Lambda^{n}(S)$ the $\mathbf{K}$-linear space generated by all the $n$-fold exterior products formed by the elements of $S$. In particular, the $\mathbf{K}$-linear space $\Lambda^{n}(\Theta)$ is closed under $\partial_{x}$ and $\partial_{y}$. For every $\lambda \in \Lambda^{n}(\Theta)$, there exists $\lambda_{L} \in \Lambda^{n}(\operatorname{pder}(L))$ s.t.

$$
\lambda\left(z_{1}, \ldots, z_{n}\right)=\lambda_{L}\left(z_{1}, \ldots, z_{n}\right)
$$

for $z_{1}, \ldots, z_{n} \in \operatorname{sol}(L)$. The exterior expression $\lambda_{L}$ can be computed by replacing each derivative appearing in $\lambda$ by its normal form w.r.t. L. Equation (3) is crucial in this paper.

\section{QUOTIENT SYSTEMS}

A factor $F$ of $L$ helps us to find a subspace of $\operatorname{sol}(L)$. Can we use $F$ to describe all the solutions of $L$ ? An answer is to use a quotient system of $L$ w.r.t. $F$, which is briefly described in [14] without proofs. We shall spell out the details.

Write $L=\left\{L_{1}, \ldots, L_{p}\right\}$, and let $F=\left\{F_{1}, \ldots, F_{q}\right\}$ be a Gröbner basis and a factor of $L$. Then

$$
L_{i}=\sum_{j=1}^{q} Q_{i j} F_{j} \quad \text { for some } Q_{i j} \in \mathbf{K}\left[\partial_{x}, \partial_{y}\right] .
$$

Since $F$ is a Gröbner basis, all the $S$-pairs of $F$

$$
\left(\frac{\delta_{a}}{f_{a}} F_{a}-\frac{\delta_{b}}{f_{b}} F_{b}\right)=\sum_{j=1}^{q} P_{a b j} F_{j}, \quad \text { for some } P_{a b j} \in \mathbf{K}\left[\partial_{x}, \partial_{y}\right]
$$

where $\delta_{a}, \delta_{b}$ are the derivatives to form the $S$-pair of $F_{a}$ and $F_{b}$, and $f_{a}, f_{b}$ are respective leading coefficients. Let $u_{0}$, $u_{1}, \ldots, u_{q}$ be differential indeterminates over $\mathbf{K}$. For an element $A \in \mathbf{K}\left[\partial_{x}, \partial_{y}\right]$, the action of $A$ on $u_{i}$ is denoted by $A\left(u_{i}\right)$ which is a linear differential polynomial in $u_{i}$. A quotient system of $L$ w.r.t. $F$ is defined to be

$$
Q=\left\{Q_{i}=0, T_{a b}=0 \mid 1 \leq i \leq p, 1 \leq a<b \leq q\right\}
$$

where

$Q_{i}=\sum_{j=1}^{q} Q_{i j}\left(u_{j}\right), T_{a b}=\left(\frac{\delta_{a}}{f_{a}}\left(u_{a}\right)-\frac{\delta_{b}}{f_{b}}\left(u_{b}\right)\right)-\sum_{j=1}^{q} P_{a b j}\left(u_{j}\right)$.
Proposition 1. Let $G\left(u_{0}, u_{1}, \ldots, u_{q}\right)$ denote the system

$$
\left\{F_{1}\left(u_{0}\right)=u_{1}, \ldots, F_{q}\left(u_{0}\right)=u_{q}\right\} .
$$

Then we have the following:

1. if $\left(v_{1}, \ldots, v_{q}\right) \in \operatorname{sol}(Q)$, then there exists $v_{0} \in \mathbf{E}$ such that $\left(v_{0}, v_{1}, \ldots, v_{q}\right) \in \operatorname{sol}(G)$, so that $v_{0} \in \operatorname{sol}(L)$;

2. if $v_{0} \in \operatorname{sol}(L)$, then $\left(F_{1}\left(v_{0}\right), \ldots, F_{q}\left(v_{0}\right)\right) \in \operatorname{sol}(Q)$;

3. $\operatorname{dim}_{\overline{\mathbf{Q}}} \operatorname{sol}(Q)+\operatorname{dim}_{\overline{\mathbf{Q}}} \operatorname{sol}(F)=d$.

Proof Let $F$ be an $n$ th-order factor. To prove the first assertion, we regard $G\left(u_{0}\right)=G\left(u_{0}, v_{1}, \ldots, v_{q}\right)$ as a differential system in $u_{0}$. Its integrability conditions $T_{a b}\left(v_{1}, \ldots, v_{q}\right)$ $(1 \leq a<b \leq q)$ vanish, since $T_{a b}$ is in $Q$. In other words, $\left\{F_{1}\left(u_{0}\right)-v_{1}, \ldots, F_{q}\left(u_{0}\right)-v_{q}\right\}$ is a linear coherent autoreduced set. Hence, $G\left(u_{0}\right)$ has a solution $v_{0}$. It follows from (4) that $v_{0} \in \operatorname{sol}(L)$. The second assertion is direct from (4) and (5). To prove the last assertion, we let $h$ be the dimension of $\operatorname{sol}(Q)$ over $\overline{\mathbf{Q}}$, and $z_{1}, \ldots, z_{n}, w_{1}$, $\ldots, w_{d-n}$ a fundamental system of $L$, in which $z_{1}, \ldots, z_{n}$ are in $\operatorname{sol}(F)$. Then the vectors $\vec{v}_{i}=\left(F_{1}\left(w_{i}\right), \ldots, F_{q}\left(w_{i}\right)\right)$, where $1 \leq i \leq(d-n)$, are nontrivial solutions of $Q$ by the second assertion. If these vectors are $\mathbf{Q}$-linearly dependent, then a nontrivial $\overline{\mathbf{Q}}$-linear combination of the $w_{i}$ is a solution of all the $F_{i}$, a contradiction to the selection of the $w_{i}$. Thus, $h \geq(d-n)$. For nonzero $\vec{v} \in \operatorname{sol}(Q)$, let $v_{0}$ satisfy $G\left(u_{0}, \vec{v}\right)$. Since $v_{0} \in \operatorname{sol}(L)$, it can be expressed as a nontrivial $\overline{\mathbf{Q}}$-linear combination of the $z_{j}$ and $w_{i}$. Applying each $F_{k}$ in $F$ to the linear combination yields that $\vec{v}$ is a Q-linear combination of the $\overrightarrow{v_{i}}$. Hence $h \leq(d-n)$.

\section{Example 3. Consider the Gröbner basis}

$$
H=\left\{\partial_{x}^{2}-\frac{y x-1}{x} \partial_{x}-\frac{y}{x}, \partial_{y}-\frac{x}{y} \partial_{x}\right\} .
$$

It has a first-order factor $\left\{F_{1}=\partial_{y}-x, F_{2}=\partial_{x}-y\right\}$. A quotient system $Q$ of $H$ w.r.t. $F$ is

$\left\{\partial_{x} u_{2}=\frac{-1}{x} u_{2}, u_{1}=\frac{x}{y} u_{2}, \partial_{x}\left(u_{1}\right)-\partial_{y}\left(u_{2}\right)=y u_{1}-x u_{2}\right\}$.

We find that $\left(u_{1}=\frac{1}{y}, u_{2}=\frac{1}{x}\right)$ is a nonzero solution of $Q$. Let $v_{0}$ be a solution of $G\left(u_{0}\right)=\left\{F_{1}\left(u_{0}\right)=\frac{1}{y}, F_{2}\left(u_{0}\right)=\frac{1}{x}\right\}$. It follows from Proposition 1 that $H$ has a fundamental system of solutions $\left\{\exp (x y), v_{0}\right\}$. If both $x$ and $y$ are positive real variables, then $v_{0}$ can be chosen as $-\exp (x y) \operatorname{Ei}(1, x y)$.

\section{SOME USEFUL FACTS}

A nonzero element $h$ of $\mathbf{E}$ is said to be hyperexponential over $\mathbf{K}$ if both $\left(\partial_{x} h\right) / h$ and $\left(\partial_{y} h\right) / h$ belong to $\mathbf{K}$. Two hyperexponential elements are said to be equivalent if their ratio belongs to $\mathbf{K}$. An easy calculation shows that the set of all hyperexponential elements over $\mathbf{K}$ is closed under multiplication and division. Hyperexponential elements play a key role in algorithms for factoring differential operators.

Proposition 2. The Gröbner basis $L$ has a first-order factor if and only if $L$ has a hyperexponential solution. 
Proof If $h$ is a hyperexponential solution of $L$, then the Gröbner basis $F=\left\{\partial_{x}-\frac{\partial_{x} h}{h}, \partial_{y}-\frac{\partial_{y} h}{h}\right\}$ is a first-order factor of $L$ over $\mathbf{K}$. Conversely, let $F=\left\{\partial_{x}-u, \partial_{y}-v\right\}$ be a first-order factor of $L$ over $\mathbf{K}$. Every nonzero solution of $F$ is hyperexponential.

The algorithm presented in [7] computes all first-order factors of a given system $L$ such as the first-order factors appearing in examples 1 and 3 .

For a $d$ th order linear ode w.r.t. $\partial_{x}$, its $n$th order right factors have leading derivative $\partial_{x}^{n}$. What is $\operatorname{lder}(F)$ if $F$ is a factor of $L$ ? The following lemma provides an answer.

Lemma 3. If $F$ is specified as above, then

$$
\operatorname{lder}(F) \subset(\operatorname{lder}(L) \cup \operatorname{pder}(L)), \operatorname{pder}(F) \subset \operatorname{pder}(L) .
$$

and, for every $\theta \in \operatorname{lder}(L)$, there exists $\delta \in \operatorname{lder}(F)$ such that $\theta$ is divisible by $\delta$.

Proof If $\delta \in \operatorname{lder}(F)$ and $\delta \notin(\operatorname{lder}(L) \cup \operatorname{pder}(L))$, then $\delta$ is divisible by some $\theta$ in $\operatorname{lder}(L)$. As each member of $L$ can be reduced to zero by $F, \theta$ is divisible by an element of $\operatorname{lder}(F)$, so is $\delta$, contradicting to the fact that $F$ is reduced. The last two assertions follow from the same argument.

Lemma 3 tells us that there are only finitely many choices for $\operatorname{lder}(F)$. As shown in Example 1, $(\operatorname{lder}(L) \cap \operatorname{lder}(F))$ may be nonempty. This point is neglected in [14].

\section{WRONSKIAN REPRESENTATIONS}

A key idea in the Beke-Schlesinger algorithm is to look for right factors whose coefficients are Wronskian-like determinants. To use this idea, we extend the notion of Wronskians. Let $F$ be a Gröbner basis in $\mathbf{K}\left[\partial_{x}, \partial_{y}\right]$ with $n$-dimensional solution space. Let $\operatorname{lder}(F)=\left\{\theta_{1}, \ldots, \theta_{k}\right\}$ and $\operatorname{pder}(F)=$ $\left\{\xi_{1}, \ldots, \xi_{n}\right\}$, where $\xi_{i}$ is lower than $\xi_{j}$ for $1 \leq i<j \leq n$. Assume that $F=\left\{F_{1}, \ldots, F_{k}\right\}$ in which each $F_{i}$ is monic with leading derivative $\theta_{i}$. We call the element $\omega_{F}=\left(\xi_{1} \wedge \cdots \wedge \xi_{n}\right)$ the Wronskian operator of $F$. It follows from (3) (replacing $L$ by $F)$ and $\Lambda^{n}(\operatorname{pder}(F))=\left\{r \omega_{F} \mid r \in \mathbf{K}\right\}$ that, for every $\lambda \in \Lambda^{n}(\Theta)$, there exists $r_{\lambda} \in \mathbf{K}$ such that

$$
\lambda\left(z_{1}, \ldots, z_{n}\right)=r_{\lambda} \omega_{F}\left(z_{1}, \ldots, z_{n}\right)
$$

for $z_{1}, \ldots, z_{n} \in \operatorname{sol}(F)$.

Lemma 4. For all $z_{1}, \ldots, z_{n} \in \operatorname{sol}(F), z_{1}, \ldots z_{n}$ are $\overline{\mathbf{Q}}$ linearly independent if and only if $\omega_{F}(\vec{z}) \neq 0$. Moreover, let $z_{1}, \ldots, z_{n}$ form a fundamental system of solutions of $F$ and denote $\left(z_{1}, \ldots z_{n}\right)$ by $\vec{z}$. Then

$$
\left(\omega_{F} \wedge \theta_{i}\right)(\vec{z}, \cdot)=\omega_{F}(\vec{z}) F_{i}, \quad i=1, \ldots, k,
$$

where $\left(\omega_{F} \wedge \theta_{i}\right)(\vec{z}, \cdot)$ means the $(n+1) \times(n+1)$ determinant

$$
\mid \begin{array}{ccccc}
\xi_{1} z_{1} & \xi_{1} z_{2} & \cdots & \xi_{1} z_{n} & \xi_{1} \\
\xi_{2} z_{1} & \xi_{2} z_{2} & \cdots & \xi_{2} z_{n} & \xi_{2} \\
\vdots & \vdots & \vdots & \vdots & \vdots \\
\xi_{n} z_{1} & \xi_{n} z_{2} & \cdots & \xi_{n} z_{n} & \xi_{n} \\
\theta_{i} z_{1} & \theta_{i} z_{2} & \cdots & \theta_{i} z_{n} & \theta_{i}
\end{array}
$$

in which derivatives are placed at the right-hand side in a product.

Proof If $z_{1}, \ldots, z_{n}$ are linearly independent over $\overline{\mathbf{Q}}$, Theorem 1 in $\left[6\right.$, page 86] implies that there exists $\lambda$ in $\Lambda^{n}(\Theta)$ such that $\lambda\left(z_{1}, \ldots, z_{n}\right) \neq 0$. The first assertion then follows from (6). The converse is true by the same theorem. The expression $\left(\omega_{F} \wedge \theta_{i}\right)(\vec{z}, \cdot)$ can be reduced to zero by $F$, because $\left(\omega_{F} \wedge \theta_{i}\right)\left(\vec{z}, z_{j}\right)=0$ for $j=1, \ldots, n$. On the other hand, expanding $\left(\omega_{F} \wedge \theta_{i}\right)(\vec{z}, \cdot)$ according to its last column yields, for all $i$ with $1 \leq i \leq k$,

$$
\left(\omega_{F} \wedge \theta_{i}\right)(\vec{z}, \cdot)=\omega_{F}(\vec{z}) \theta_{i}+\sum_{j=1}^{d} \underbrace{(-1)^{d+j+1}\left(\eta_{j} \wedge \theta_{i}\right)(\vec{z})}_{w_{i j}} \xi_{j},
$$

where $\eta_{j}=\xi_{1} \wedge \cdots \wedge \xi_{j-1} \wedge \xi_{j+1} \wedge \cdots \wedge \xi_{n}$. It follows that $\left(\left(\omega_{F} \wedge \theta_{i}\right)(\vec{z}, \cdot)-\omega_{F}(\vec{z}) F_{i}\right)$ equals zero.

We call $\left\{\left(\omega_{F} \wedge \theta_{1}\right)(\vec{z}, \cdot), \ldots,\left(\omega_{F} \wedge \theta_{k}\right)(\vec{z}, \cdot)\right\}$ a Wronskian representation of $F$. Any two Wronskian representations of $F$ can only differ by a multiplicative constant in $\overline{\mathbf{Q}}$, because any two sets of fundamental solutions of $F$ can be transformed from one to the other by a matrix over $\overline{\mathbf{Q}}$.

Corollary 5. The Wronskian representation of $F$ is a reduced Gröbner basis over $\mathbf{K}<z_{1}, \ldots, z_{n}>$, the differential field generated by $z_{1}, \ldots, z_{n}$ over $\mathbf{K}$.

ExAmple 4. The Wronskian representation of a Gröbner basis $F$ with $\operatorname{lder}(F)=\left\{\partial_{x}^{2}, \partial_{y}\right\}$ is

$$
\begin{aligned}
& \left\{\left(1 \wedge \partial_{x}\right)(\vec{z}) \partial_{x}^{2}-\left(1 \wedge \partial_{x}^{2}\right)(\vec{z}) \partial_{x}+\left(\partial_{x} \wedge \partial_{x}^{2}\right)(\vec{z}),\right. \\
& \left.\left(1 \wedge \partial_{x}\right)(\vec{z}) \partial_{y}-\left(1 \wedge \partial_{y}\right)(\vec{z}) \partial_{x}+\left(\partial_{x} \wedge \partial_{y}\right)(\vec{z})\right\}
\end{aligned}
$$

where $\vec{z}=\left(z_{1}, z_{2}\right)$ and $z_{1}, z_{2}$ form a fundamental system of solutions of $F$. The Wronskian representation of $F$ with leading derivatives $\partial_{y}^{2}$ and $\partial_{x}$ is

$$
\begin{aligned}
& \left\{\left(1 \wedge \partial_{y}\right)(\vec{z}) \partial_{y}^{2}-\left(1 \wedge \partial_{y}^{2}\right)(\vec{z}) \partial_{y}+\left(\partial_{y} \wedge \partial_{y}^{2}\right)(\vec{z}),\right. \\
& \left.\left(1 \wedge \partial_{y}\right)(\vec{z}) \partial_{x}+\left(\partial_{y} \wedge \partial_{x}\right)(\vec{z})\right\}
\end{aligned}
$$

since $\left(1 \wedge \partial_{x}\right)(\vec{z})$ has to be zero, for, otherwise, the representation would not be reduced, a contradiction to Corollary 5 .

The next proposition reveals that the coefficients $w_{i j}$ are quite simple although they may be outside $\mathbf{K}$.

Proposition 6. Let $F$ be a Gröbner basis in $\mathbf{K}\left[\partial_{x}, \partial_{y}\right]$, $z_{1}, \ldots, z_{n}$ form a fundamental system of solutions of $F$, and denote $\left(z_{1}, \ldots, z_{n}\right)$ by $\vec{z}$. Then $\omega_{F}(\vec{z})$ is hyperexponential over $\mathbf{K}$. Furthermore, for all $\lambda \in \Lambda^{n}(\Theta), \lambda(\vec{z})$ is either zero or hyperexponential over $\mathbf{K}$, so is every $w_{i j}$ given in (7).

Proof Lemma 4 implies that $\omega_{F}(\vec{z})$ is nonzero. It follows from (6) that the ratio of $\partial_{x} \omega_{F}(\vec{z})$ and $\omega_{F}(\vec{z})$ belongs to $\mathbf{K}$. The same conclusion holds for the ratio of $\partial_{y} \omega_{F}(\vec{z})$ and $\omega_{F}(\vec{z})$. Hence, $\omega_{F}(\vec{z})$ is hyperexponential over $\mathbf{K}$. Consequently, any nonzero $\lambda(\vec{z})$ is hyperexponential by $(6)$.

We show how to compute $\omega_{F}(\vec{z})$ and the coefficients $w_{i j}$ in the next section. 


\section{ASSOCIATED SYSTEMS}

In this section we generalize the notion of associated equations for factoring linear ode's. As in the previous sections, let $n$ be an integer with $1 \leq n<d$. We want to regard every element of $\Lambda^{n}(\Theta)$ as a function on $\operatorname{sol}(L)^{n}$. Two elements of $\Lambda^{n}(\Theta)$ are said to be equivalent if they are identical (as functions) on $\operatorname{sol}(L)^{n}$. For an element $\lambda$ of $\Lambda^{n}(\Theta)$, its equivalence class is denoted by $\bar{\lambda}$. It is easy to verify that the equivalence relation is compatible with linear operations and differentiations on $\Lambda^{n}(\Theta)$. The $\mathbf{K}$-linear space consisting of the equivalence classes is called the $n$th Beke space relative to $L$, and denoted by $B_{n}$ when $L$ is clear from the context. From (3) it follows that each equivalence class contains an element of $\Lambda^{n}(\operatorname{pder}(L))$. Consequently, $B_{n}$ can be $\mathbf{K}$-linearly generated by the elements in form

$$
\overline{\left(\theta_{1} \wedge \theta_{2} \wedge \cdots \wedge \theta_{n}\right)}
$$

where the $\theta_{i}$ belong to $\operatorname{pder}(L)$ and $\theta_{i}$ is lower than $\theta_{j}$ for all $i, j$ with $1 \leq i<j \leq n$. The elements in (10) are called canonical generators of $B_{n}$. They are not necessarily K-linearly independent. The following lemma is evident.

Lemma 7. The nth Beke space $B_{n}$ is of dimension less than or equal to $\left(\begin{array}{l}d \\ n\end{array}\right)$ and closed under differentiation.

ExAmple 5. If $\operatorname{lder}(L)=\left\{\partial_{x}^{2}, \partial_{x} \partial_{y}, \partial_{y}^{2}\right\}$, then $\operatorname{pder}(L)=$ $\left\{1, \partial_{x}, \partial_{y}\right\}$. The second Beke space $B_{2}$ relative to $L$ is generated by $\overline{\left(1 \wedge \partial_{x}\right)}, \overline{\left(1 \wedge \partial_{y}\right)}$, and $\overline{\left(\partial_{x} \wedge \partial_{y}\right)}$.

Set $m=\left(\begin{array}{l}d \\ n\end{array}\right)$. For an element $\bar{\lambda}$ of $B_{n}$, the ideal consisting of all annihilators of $\bar{\lambda}$ in $\mathbf{K}\left[\partial_{x}, \partial_{y}\right]$ is denoted by $\operatorname{ann}(\bar{\lambda})$. It follows from Lemma 7 that the dimension of the solution space of $\operatorname{ann}(\bar{\lambda})$ is not greater than $m$. A finite subset of $\operatorname{ann}(\bar{\lambda})$ with finite-dimensional solution space is called a system associated with $\bar{\lambda}$. The following method computes an associated system by linear algebra and differential reduction. Lemma 7 implies that both $\bar{\lambda}, \partial_{x} \bar{\lambda}, \ldots$, $\partial_{x}^{m} \bar{\lambda}$ and $\bar{\lambda}, \partial_{y} \bar{\lambda}, \ldots, \partial_{y}^{m} \bar{\lambda}$ are K-linearly dependent. Suppose that $p$ and $q$ are smallest nonnegative integers such that $\partial_{x}^{p} \bar{\lambda}+\sum_{i=0}^{p-1} f_{i} \partial_{x}^{i} \bar{\lambda}=0$ and $\partial_{y}^{q} \bar{\lambda}+\sum_{j=0}^{q-1} g_{j} \partial_{y}^{j} \bar{\lambda}=0$. where $f_{p-1}, \ldots, f_{0}, g_{q-1}, \ldots, g_{0} \in \mathbf{K}$. We find the system

$$
\left\{\partial_{x}^{p}+\sum_{i=0}^{p-1} f_{i} \partial_{x}^{i}, \partial_{y}^{q}+\sum_{j=0}^{q-1} g_{j} \partial_{y}^{j}\right\}
$$

annihilating $\bar{\lambda}$. The solution space of (11) is of finite dimension, because parametric derivatives of a Gröbner basis for the ideal generated by (11) are contained in

$$
D_{\lambda}=\left\{\partial_{x}^{i} \partial_{y}^{j} \mid 0 \leq i<p, 0 \leq j<q\right\} .
$$

Hence, (11) is a system associated with $\bar{\lambda}$. Considering all possible K-linear combinations of $(m+1)$ elements of $D_{\lambda}$, we may obtain an associated system with $m$-dimensional solution space (see the proof of Lemma 1 in [14]). With the help of noncommutative elimination techniques (see e.g. [3]), one may possibly find associated systems with lower orders.

To factor 0-dim ideals, we need systems associated with the canonical generators. The following method for constructing these systems in form (11) is an easy generalization of the method described in [2]. Let $\vec{b}_{n}$ be the $m$-dimensional vector consisting of the canonical generators of $B_{n}$. Differentiating this vector and using (3), we obtain two $(m \times m)$ matrices $M_{n}$ and $N_{n}$ over $\mathbf{K}$ such that

$$
\partial_{x} \vec{b}_{n}=\vec{b}_{n} M_{n} \text { and } \partial_{y} \vec{b}_{n}=\vec{b}_{n} N_{n} .
$$

Set both $M_{n, 0}$ and $N_{n, 0}$ to be the $(m \times m)$ unit matrix, and set $M_{n, 1}=M_{n}$ and $N_{n, 1}=N_{n}$. Define:

$M_{n, i}=M_{n} M_{n, i-1}+\partial_{x} M_{n, i-1}, N_{n, i}=N_{n} N_{n, i-1}+\partial_{y} N_{n, i-1}$,

where $i \geq 2$, respectively. An easy induction leads to

$$
\partial_{x}^{i} \vec{b}_{n}=\vec{b}_{n} M_{n, i} \quad \text { and } \quad \partial_{y}^{i} \vec{b}_{n}=\vec{b}_{n} M_{n, i} \quad(i \geq 0) .
$$

For $j=1, \ldots, m$, let $\vec{g}_{n, j}$ and $\vec{h}_{n, j}$ be $(m+1)$-dimensional vectors whose $k$ th vector is the $(k-1)$ th derivatives of the $j$ th component of $\vec{b}_{n}$ w.r.t. $x$ and $y$, respectively. Then

$$
\vec{g}_{n, j}=\vec{b}_{n} U_{n, j} \quad \text { and } \quad \vec{h}_{n, j}=\vec{b}_{n} V_{n, j}
$$

where the $k$ th column of the $(m \times(m+1))$ matrix $U_{n, j}\left(V_{n, j}\right)$ is the $j$ th column of $M_{n, k-1}\left(N_{n, k-1}\right)$, for $k=1, \ldots, m+1$. Since both $U_{n, j}$ and $V_{n, j}$ have more columns than rows, their columns are K-linearly dependent. From these linear relations we can extract a system associated with the $j$ th component of $\vec{b}_{n}$. Briefly, the systems associated with the canonical generators of $B_{n}$ can be constructed by solving $2 m$ $\mathbf{K}$-linear homogeneous algebraic systems whose coefficient matrices are $U_{n, j}$ and $V_{n, j}$ for $j=1, \ldots, m$.

ExAmPLE 6 . Let $L$ be given in (1) and $n=2$. Write

$$
L=\left\{\partial_{x}^{2}+l_{1}, \partial_{x} \partial_{y}+l_{2}, \partial_{y}^{2}+l_{3}\right\}
$$

where the $l_{i}$ 's are $\mathbf{K}$-linear combinations of $1, \partial_{x}$, and $\partial_{y}$. Three canonical generators of $B_{2}$ are

$$
b_{1}=\overline{\left(1 \wedge \partial_{x}\right)}, b_{2}=\overline{\left(1 \wedge \partial_{y}\right)}, b_{3}=\overline{\left(\partial_{x} \wedge \partial_{y}\right)} .
$$

By differentiation we get

$$
\begin{gathered}
\partial_{x} b_{1}=\overline{1 \wedge \partial_{x}^{2}}=-\overline{1 \wedge l_{1}} . \\
\partial_{x} b_{2}=\overline{\partial_{x} \wedge \partial_{y}}+\overline{1 \wedge \partial_{y}^{2}}=\overline{\partial_{x} \wedge \partial_{y}}-\overline{1 \wedge l_{3}} . \\
\partial_{x} b_{3}=\overline{\partial_{x}^{2} \wedge \partial_{y}}+\overline{\partial_{x} \wedge \partial_{x} \partial_{y}}=-\overline{l_{1} \wedge \partial_{y}}-\overline{\partial_{x} \wedge l_{2}} .
\end{gathered}
$$

Substituting concrete expressions of the $l_{i}$ yields

$$
M_{2}=\left(\begin{array}{ccc}
\frac{x y-2+x y}{4 x} & \frac{2+x y}{4 y} & \frac{-1}{4} \\
\frac{-y^{2}}{4 x} & \frac{-y}{4} & \frac{y}{4 x} \\
0 & 1 & \frac{-1}{2 x}
\end{array}\right)
$$

as described in (12). In the same vein, we have

$$
N_{2}=\left(\begin{array}{ccc}
\frac{2+x y}{4 y} & \frac{x(x y-2)}{4 y^{2}} & \frac{-x}{4 y} \\
\frac{-y}{4} & \frac{-x}{4} & \frac{1}{4} \\
-1 & 0 & \frac{1}{2 y}
\end{array}\right)
$$

Using (13) to find $M_{22}, M_{23}, N_{22}$ and $N_{23}$, and constructing $U_{2 j}$ and $V_{2 j}$ for $j=1,2,3$, we find that the systems associated with $b_{1}, b_{2}$ and $b_{3}$ are, respectively,

$$
\begin{aligned}
& \left\{\partial_{x}^{3}+\frac{3}{x} \partial_{x}^{2}-\frac{-3+x y}{4 x^{2}} \partial_{x}-\frac{y}{8 x^{2}},\right. \\
& \left.\quad \partial_{y}^{3}-\frac{2 x y-6}{y(x y-6)} \partial_{y}^{2}-\frac{42-23 x y+x^{2} y^{2}}{4 y^{2}(x y-6)} \partial_{y}+\frac{72-30 x y+x^{2} y^{2}}{8 y^{3}(x y-6)}\right\},
\end{aligned}
$$




$$
\begin{aligned}
& \left\{\partial_{x}^{3}+\frac{2}{x(2+x y)} \partial_{x}^{2}-\frac{x y+1}{4 x^{2}} \partial_{x}+\frac{y^{2}}{8 x(2+x y)},\right. \\
& \partial_{y}^{3}+\frac{x y-6}{y(-2+x y)} \partial_{y}^{2}-\frac{-12+x^{3} y^{3}-12 x y-x^{2} y^{2}}{4 y^{2}\left(4-4 x y+x^{2} y^{2}\right)} \partial_{y} \\
& \left.\quad-\frac{x^{2}(-10+x y)}{8 y\left(4-4 x y+x^{2} y^{2}\right)}\right\},
\end{aligned}
$$

and

$$
\left\{\partial_{x}^{3}+\frac{15+x y}{6 x} \partial_{x}^{2}-\frac{y^{2}}{24 x}, \partial_{y}^{2}-\frac{1}{2 y} \partial_{y}-\frac{x y-2}{4 y^{2}}\right\} .
$$

\section{IDEA ABOUT FACTORIZATION}

As before, we let $F$ be a Gröbner basis in $\mathbf{K}\left[\partial_{x}, \partial_{y}\right]$ such that $\operatorname{sol}(F) \subset \operatorname{sol}(L)$ and $\operatorname{dim}_{\overline{\mathbf{Q}}} \operatorname{sol}(F)=n$, where $1 \leq n<d$. Let $\vec{z}$ be the vector $\left(z_{1}, \ldots, z_{n}\right)$ where $z_{1}, z_{2}, \ldots, z_{n}$ form a fundamental system of solutions of $F$.

\subsection{Candidates for canonical generators}

Let $b_{1}, \ldots, b_{m}$ be the canonical generators of $B_{n}$ with respective associated systems $A_{1}, \ldots, A_{m}$. Lemma 3 implies that $\bar{\omega}_{F}$ is a canonical generator of $B_{n}$. Hence, the function value $\omega_{F}(\vec{z})$ is a hyperexponential solution of its associated systems by the first assertion of Proposition 6. For every $b_{i}$, the function value $b_{i}(\vec{z})$ is a solution of $A_{i}$, which is either 0 or hyperexponential by the second assertion of Proposition 6. Applying the algorithm in [7], we can find all possible candidates for $b_{1}(\vec{z}), \ldots, b_{m}(\vec{z})$.

Example 7. Consider the system $L$ given in (1). According to the associated systems presented in Example 6, we find the candidates of $b_{1}(\vec{z}), b_{2}(\vec{z})$, and $b_{3}(\vec{z})$ are

$$
\left\{\frac{c_{1} y}{\sqrt{x y}} \mid c_{1} \in \overline{\mathbf{Q}}\right\},\left\{\frac{c_{2} x}{\sqrt{x y}} \mid c_{2} \in \overline{\mathbf{Q}}\right\} \text {, and }\{0\},
$$

respectively.

\subsection{Candidates for Wronskian coefficients}

\section{Assume that}

$$
\operatorname{lder}(F)=\left\{\theta_{1}, \ldots, \theta_{k}\right\} \text { and } \operatorname{pder}(F)=\left\{\xi_{1}, \ldots, \xi_{n}\right\} .
$$

The Wronskian coefficients of $F$

$$
w_{i j}=(-1)^{d+j+1}\left(\eta_{j} \wedge \theta_{i}\right)(\vec{z})
$$

given in (7) are $\mathbf{K}$-linear combinations of the $b_{i}(\vec{z})$ 's, because $\overline{\eta_{j} \wedge \theta_{i}} \in B_{n}$ and $z_{1}, \ldots, z_{n} \in \operatorname{sol}(L)$. Hence, we can obtain all candidates for the $w_{i j}$ 's as long as the candidates for the $b_{i}(\vec{z})$ 's are known.

Example 8. Again, let $L$ be given in (1). The Wronskian representation of $F$ given in (8) is

$$
\begin{aligned}
& \left\{\left(1 \wedge \partial_{x}\right)(\vec{z}) \partial_{x}^{2}-\left(1 \wedge \partial_{x}^{2}\right)(\vec{z}) \partial_{x}+\left(\partial_{x} \wedge \partial_{x}^{2}\right)(\vec{z})\right. \\
& \left.\left(1 \wedge \partial_{x}\right)(\vec{z}) \partial_{y}-\left(1 \wedge \partial_{y}\right)(\vec{z}) \partial_{x}+\left(\partial_{x} \wedge \partial_{y}\right)(\vec{z})\right\}
\end{aligned}
$$

Let $b_{1}, b_{2}, b_{3}$ be the same as in Example 7 . In $B_{2}$ we have

$$
\overline{1 \wedge \partial_{x}^{2}}=-\frac{2-x y}{4 x} b_{1}-\frac{y^{2}}{4 x} b_{2}, \overline{\partial_{x} \wedge \partial_{x}^{2}}=-\frac{y}{4 x} b_{1}-\frac{y^{2}}{4 x} b_{3} .
$$

As the components $z_{1}$ and $z_{2}$ of $\vec{z}$ belong to $\operatorname{sol}(L)$, the above equalities and Example 7 imply that the Wronskian representation of $F$ must be in form

$$
\begin{gathered}
\left\{\frac{c_{1} y}{\sqrt{x y}} \partial_{x}^{2}+\left(\frac{2-x y}{4 x} \frac{c_{1} y}{\sqrt{x y}}+\frac{y^{2}}{4 x} \frac{c_{2} x}{\sqrt{x y}}\right) \partial_{x}-\frac{y}{4 x} \frac{c_{1} y}{\sqrt{x y}},\right. \\
\left.\frac{c_{1} y}{\sqrt{x y}} \partial_{y}-\frac{c_{2} x}{\sqrt{x y}} \partial_{x}\right\}
\end{gathered}
$$

where $c_{1}, c_{2} \in \overline{\mathbf{Q}}$ with $c_{1} \neq 0$.

By computing hyperexponential solutions of systems associated with the canonical generators and performing linear operations in $B_{n}$, we can get all possible candidates for the Wronskian representation of $F$. There are only finitely many such candidates, each of which may involve a finite number of unspecified constants in $\overline{\mathbf{Q}}$. This is due to the structure of hyperexponential solutions of $L$ (see [7, Theorem 3.2]).

\subsection{Determine coefficients}

It remains to determine which candidate leads to a factor of $L$. A monic associate of a candidate is the set consisting of monic associates of all elements in the candidate. According to (6), the ratio of $\omega_{F}(\vec{z})$ and any coefficient appearing in some equation of a candidate belongs to $\mathbf{K}$. Therefore, we exclude those candidates whose monic associates do not belong to $\mathbf{K}\left[\partial_{x}, \partial_{y}\right]$. Let $M$ be a monic associate of a candidate contained in $\mathbf{K}\left[\partial_{x}, \partial_{y}\right]$. The system $M$ is a factor of $L$ with $\operatorname{dim}_{\overline{\mathbf{Q}}} \operatorname{sol}(M)=n$ if and only if

- $M$ is a Gröbner basis with $\operatorname{lder}(M)=\left\{\theta_{1}, \ldots, \theta_{k}\right\}$.

- For each element of $L$, its normal form w.r.t. $M$ is zero.

Example 9. The monic associate of (16) is

$$
\left\{\partial_{x}^{2}+\left(\frac{2-x y}{4 x}+\frac{c y}{4}\right) \partial_{x}-\frac{y}{4 x}, \partial_{y}-\frac{c x}{y} \partial_{x}\right\}
$$

where $c=c_{2} / c_{1}$. This system has leading derivatives $\partial_{x}^{2}$ and $\partial_{y}$. A simple calculation shows that (17) is a Gröbner basis if and only if $c=1$. Setting $c=1$ and computing the normal form of every element of $L$ given in (1) w.r.t. (17), we confirm that $L$ has a factor $F$ as given in Example 1 .

EXAmPLE 10. Let us determine factors of $L$ given in (1) with leading derivatives $\partial_{y}^{2}$ and $\partial_{x}$. Assume that $G$ is such a factor with a set of fundamental solutions $z_{1}$ and $z_{2}$, and denote $\left(z_{1}, z_{2}\right)$ by $\vec{z}$. In this case the Wronskian operator of $G$ becomes $\omega_{G}=\left(1 \wedge \partial_{y}\right)$. The candidates for $\omega_{G}(\vec{z})$ are $\left\{\frac{c_{2} x}{\sqrt{x y}} \mid c_{2} \in \overline{\mathbf{Q}}, c_{2} \neq 0\right\}$ as given in (15). As shown in (9), the Wronskian representation of $G$ is

$$
\begin{aligned}
& \left\{\left(1 \wedge \partial_{y}\right)(\vec{z}) \partial_{y}^{2}-\left(1 \wedge \partial_{y}^{2}\right)(\vec{z}) \partial_{y}+\left(\partial_{y} \wedge \partial_{y}^{2}\right)(\vec{z})\right. \\
& \left.\left(1 \wedge \partial_{y}\right)(\vec{z}) \partial_{x}+\left(\partial_{y} \wedge \partial_{x}\right)(\vec{z})\right\}
\end{aligned}
$$

The candidates for the Wronskian representation are

$$
\left\{\frac{c_{2} x}{\sqrt{x y}} \partial_{y}^{2}+\frac{c_{2} x^{2}}{4 \sqrt{x y}} \partial_{y}-\frac{x}{4 y} \frac{c_{2} x}{\sqrt{x y}}, \frac{c_{2} x}{\sqrt{x y}} \partial_{x}\right\}
$$

with monic associate $M=\left\{\partial_{y}^{2}+\left(\frac{x}{4}\right) \partial_{y}-\frac{x}{4 y}, \partial_{x}\right\}$. But $M$ is not a Gröbner basis. So $F$ has no factor with leading 
derivatives $\partial_{y}^{2}$ and $\partial_{x}$. Hence, (1) has only one second-order factor as given in Example 9 .

\section{FACTORIZATION ALGORITHM}

For simplicity, we describe an algorithm for finding $n$ thorder factors $F$ of $L$ under the assumption that $\operatorname{lder}(F)$ is given. It is easy to adjust the algorithm to compute all factors of $L$ by Lemma 3 .

Algorithm F Given a Gröbner basis $L$ with $d$-dimensional solution space, and $\Delta \subset(\operatorname{lder}(L) \cup \operatorname{pder}(L))$ whose elements are mutually reduced, compute all factors $F$ of $L$ with $\operatorname{lder}(F)=\Delta$.

1. [compute $\operatorname{pder}(F)]$ Find $\Delta^{-} \subset \Theta$ consisting of all derivatives not divisible by any elements of $\Delta$. If $\left|\Delta^{-}\right| \geq d$, exit [no such factors exist]. Set $n=\left|\Delta^{-}\right|$and $m=\left(\begin{array}{l}d \\ n\end{array}\right)$.

2. [candidates for the Wronskian] Construct the system $A_{1}$ associated with $\omega_{F}$, and compute hyperexponential solutions of $A_{1}$. If no hyperexponential solution is found, exit [no such factors exist]. Organize the solutions as equivalence classes:

$$
\begin{gathered}
\left\{h_{11}=p_{11} \exp \left(\int\left(f_{11} \mathrm{~d} x+g_{11} \mathrm{~d} y\right)\right), \cdots,\right. \\
h_{1 k}=p_{1 k} \exp \left(\int\left(f_{1 k} \mathrm{~d} x+g_{1 k} \mathrm{~d} y\right)\right\}
\end{gathered}
$$

where the $f_{1 i}$ and $g_{1 i}$ are in $\mathbf{K}$, and the $p_{1 i}$ are polynomials whose coefficients are elements of $\overline{\mathbf{Q}}$ and unspecified constants (see [7, Theorem 3.2]).

3. [candidates for other canonical generators] Construct the systems $A_{2}, \ldots, A_{m}$ associated with other canonical generators, and compute their hyperexponential solutions equivalent to some $h_{1 i}(1 \leq i \leq k)$. Set $h_{j i}$ to be the hyperexponential solution of $A_{j}$ equivalent to $h_{1 i}$ if such a solution exists. Otherwise, set $h_{j i}$ to be zero. Let

$$
H=\left\{\left(h_{11}, h_{21}, \ldots, h_{m 1}\right), \ldots,\left(h_{1 k}, h_{2 k}, \ldots, h_{m k}\right)\right\}
$$

where the $h_{1 i}$ are in (18), and the $h_{j i}$ with $j>1$ are either zero or hyperexponential elements equivalent to $h_{1 i}$.

4. [candidates for factors] Construct the Wronskian representation defined by $\Delta$. Construct the matrix transforming the canonical generators to the Wronskian coefficients. Use this matrix and the elements of $H$ to get all rational monic associates $\left\{F_{1}, \ldots, F_{k}\right\}$ of the candidates for factors.

5. [true factors] Check if each $F_{i}$ satisfies the two conditions given in Section 7.3, and solve algebraic equations in unspecified constants when necessary. Return the true factors.

A few words need to be said about Algorithm F. The first step is clear. The second step is a direct application of the algorithm in [7] whose outputs are distinct equivalence classes of hyperexponential solutions (after a formal integration). If no hyperexponential solution is found, then factors with leading derivatives $\Delta$ do not exist by Proposition 6 . In the third step, (6) implies that we need only hyperexponential solutions equivalent to some $h_{1 i}$. Since these solutions belong to one equivalence class, all of them can be expressed as $q_{i} h_{1 i}$, where $q_{i}$ is a polynomial in $x$ and $y$ whose coefficients are elements of $\overline{\mathbf{Q}}$ and unspecified constants. Thus, $H$ contains at most $k$ elements. Finding these solutions amounts to computing rational solutions of some 0-dim ideals, which is easier than computing all hyperexponential solutions of other associated systems. This technique is introduced in [2] for the ode case, and is extended to the pde case in [14]. The last two steps have been explained in Sections 7.2 and 7.3, respectively.

The techniques to use invertible matrices to avoid finding hyperexponential solutions in [2] can also be generalized to our case, in which the matrices $U_{n j}$ and $V_{n j}$ in (14) play the same role as $A_{S}$ in [2]. The results in Remark 2 also help to detect factors, as will be shown in the next example.

Example 11. Apply Algorithm $\mathbf{F}$ to find all factors of $L$ :

$$
\begin{gathered}
\left\{\partial_{x}^{3}+\frac{x^{3}-3 x}{x^{2}-1} \partial_{x}^{2}, \partial_{y} \partial_{x}+\frac{3 y x^{2}}{x^{2}-1} \partial_{x}^{2}+3 y x \partial_{x}+x \partial_{y},\right. \\
\left.\partial_{y}^{2}+\frac{9 y^{2} x+3 x+y}{x^{2}-1} \partial_{x}^{2}+3 y \partial_{y}+\left(3+9 y^{2}\right) \partial_{x}\right\} .
\end{gathered}
$$

All the $c$ with subscripts are hereafter unspecified constants.

We start to compute first-order factors. By Proposition 2 the algorithm in [7] yields all the first-order factors $F_{1}$ :

$$
\left\{\partial_{x}-\frac{2 c_{2}}{2 c_{1}+2 c_{2} x-3 y^{2} c_{2}}, \partial_{y}+\frac{c_{2} y}{2 c_{1}+2 c_{2} x-3 y^{2} c_{2}}\right\} .
$$

To find second-order factors, we need canonical generators of the second Beke space, which are:

$$
\begin{gathered}
b_{1}=\overline{1 \wedge \partial_{x}}, \quad b_{2}=\overline{1 \wedge \partial_{y}}, \quad b_{3}=\overline{1 \wedge \partial_{x}^{2}}, \\
b_{4}=\overline{\partial_{x} \wedge \partial_{y}}, \quad b_{5}=\overline{\partial_{x} \wedge \partial_{x}^{2}}, \quad b_{6}=\overline{\partial_{y} \wedge \partial_{x}^{2}} .
\end{gathered}
$$

We do not display their associated systems, for they are quite large. Note that associated systems only depend on $L$.

First, we compute factors with leading derivatives $\left\{\partial_{x}^{2}, \partial_{y}\right\}$. Step 1 yields $\Delta^{-}=\left\{1, \partial_{x}\right\}$. Hence, the Wronskian operator is $b_{1}$. Step 2 finds candidates

$$
\left\{c_{11},\left(c_{11}+c_{12} x+c_{13} x^{2}\right) \exp \left(\frac{-x^{2}}{2}\right)\right\}
$$

for $b_{1}$. Step 3 finds candidates $\left(c_{11}, c_{21}, 0,0,0,0\right)$ and

$$
\begin{array}{ll}
\left(c_{11}+c_{12} x+c_{13} x^{2}\right) u, & \left(c_{21}+c_{22} x+c_{23} y+c_{24} x y\right) u, \\
& 0,0,0,0)
\end{array}
$$

where $u=\exp \left(\frac{-x^{2}}{2}\right)$, for canonical generators. Step 4 gets the Wronskian representation

$$
\left\{b_{1} \partial_{x}^{2}-b_{3} \partial_{x}+b_{5}, b_{1} \partial_{y}-b_{2} \partial_{x}+b_{4}\right\}
$$

and, thus, yields two candidates

$$
\left\{\partial_{x}^{2}, \partial_{y}+c_{21} \partial_{x}\right\},\left\{\partial_{x}^{2}, \partial_{y}+\frac{c_{21}+c_{22} x+c_{23} y+c_{24} x y}{c_{11}+c_{12} x+c_{13} x^{2}} \partial_{x}\right\}
$$

for factors. In the last step we obtain a factor

$$
F_{21}:\left\{\partial_{x}^{2}, \partial_{y}+3 y \partial_{x}\right\}
$$

from the second candidate. 
Next, we compute factors with leading derivatives $\partial_{y}^{2}$ and $\partial_{x}$. The Wronskian operator then becomes $b_{2}$. Step 2 yields six candidates

$$
\begin{aligned}
& \left\{c_{21},\left(c_{21}+c_{22} x+c_{23} y+c_{24} x y\right) \exp \left(\frac{-x^{2}}{2}\right),\right. \\
& \quad c_{21} \exp \left(\frac{-3 y^{2}}{2}-x^{2}\right),\left(c_{21}+c_{22} x\right) \exp \left(\frac{-3 y^{2}}{2}+\frac{-x^{2}}{2}\right), \\
& \left.\quad c_{21} \exp \left(-x^{2}\right), c_{21} \exp \left(\frac{-3 y^{2}}{2}\right)\right\}
\end{aligned}
$$

for $b_{2}$. Note that $b_{1}$ has to be zero in this case, for otherwise, we would not have factors with desired leading derivatives. Step 3 gives six candidates for $b_{2}$. Deleting repetitions, we find that three of them are left. They are

$$
\left(0, c_{21}, 0,0,0,0\right),\left(0, c_{21} u, 0, c_{41} x u, 0, c_{61}\left(1-x^{2}\right) u\right)
$$

where $u=\exp \left(\frac{-3}{2} y^{2}-x^{2}\right)$, and

$$
\left(0,\left(c_{21}+c_{22} x\right) v, 0,\left(c_{41}+c_{42} x\right) v, 0, c_{61}\left(1-x^{2}\right) v\right)
$$

where $v=\exp \left(\frac{-3}{2} y^{2}-\frac{1}{2} x^{2}\right)$. In Step 4 we find the Wronskian representation

$$
\left\{b_{2} \partial_{y}^{2}-\overline{\left(1 \wedge \partial_{y}^{2}\right)} \partial_{y}+\overline{\left(\partial_{y} \wedge \partial_{y}^{2}\right)}, b_{2} \partial_{x}-b_{4}\right\},
$$

and transformations $\overline{\left(1 \wedge \partial_{y}^{2}\right)}=r b_{3}-3 y b_{2}-\left(9 y^{2}-3\right) b_{1}$ and $\overline{\left(\partial_{y} \wedge \partial_{y}^{2}\right)}=r b_{6}+\left(9 y^{2}+3\right) b_{4}$, where $r=\frac{9 y^{2} x+3 x+y}{1-x^{2}}$. They lead to two candidates for factors. Step 5 yields a factor

$$
F_{22}:\left\{\partial_{y}^{2}+3 y \partial_{y}+y, \partial_{x}+x\right\} .
$$

We have found all second-order factors of $L$.

Instead of algorithm $\mathbf{F}$ we use the results in Remark 2 to find all third-order factors. A straightforward calculation shows that every factor $F_{11}$ obtained from a specialization of unspecified constants in $F_{1}$ satisfies $\operatorname{sol}\left(F_{11}\right) \subset \operatorname{sol}\left(F_{21}\right)$, and that $\operatorname{sol}\left(F_{21}\right) \cap \operatorname{sol}\left(F_{22}\right)=\{0\}$. Therefore, $F_{22}$ is irreducible. It follows that

$$
\{0\} \subset \operatorname{sol}\left(F_{11}\right) \subset \operatorname{sol}\left(F_{21}\right) \subset \operatorname{sol}(L)
$$

is a maximal chain, for, otherwise, there would be a chain starting with $\{0\} \subset \operatorname{sol}\left(F_{22}\right)$ and having length $>4$. Hence, any third-order factor $F_{3}$ must satisfy $\operatorname{sol}\left(F_{22}\right) \subset \operatorname{sol}\left(F_{3}\right)$. The dimension formula from linear algebra shows

$$
\operatorname{dim}_{\overline{\mathbf{Q}}}\left(\operatorname{sol}\left(F_{3}\right)+\operatorname{sol}\left(F_{21}\right)\right)+\operatorname{dim}_{\overline{\mathbf{Q}}}\left(\operatorname{sol}\left(F_{3}\right) \cap \operatorname{sol}\left(F_{21}\right)\right)=5 .
$$

We conclude that $\left(\operatorname{sol}\left(F_{3}\right) \cap \operatorname{sol}\left(F_{21}\right)\right)$ is of dimension one over $\overline{\mathbf{Q}}$, because $\left(\operatorname{sol}\left(F_{3}\right)+\operatorname{sol}\left(F_{21}\right)\right)$ is contained in $\operatorname{sol}(L)$ whose dimension is four over $\overline{\mathbf{Q}}$. It follows that a third-order factor has a first-order factor, which is an instance of $F_{1}$. Hence, the ideal generated by a third-order factor is the intersection of the respective ideals generated by $F_{22}$ and an instance of $F_{1}$. All third-order factors can then be computed by standard techniques from Gröbner basis arsenal.

\section{CONCLUDING REMARKS}

The results of this article are a first step toward generalizing the factorization theory of linear ode's to pde's. The limitation to two independent variables is justified by its applications to the symmetry analysis on nonlinear ode's. A more complete theory will deal with any number of dependent and independent variables. In this way it will be possible to generalize Loewy's decomposition of linear ode's to systems of linear pde's with a finite-dimensional solution space.
The complexity of Algorithm F is yet unknown, partly because the algorithm for finding hyperexponential solutions of a 0-dim ideal in [7] has not been subject to a thorough complexity analysis. Many steps in Algorithm F need to be refined to obtain a reasonably efficient implementation.

\section{REFERENCES}

[1] E. Beke. Die Irreduzibilität der homogenen linearen Differentialgleichungen. Mathematische Annalen, 45:278-294, 1894.

[2] M. Bronstein. An improved algorithm for factoring linear ordinary differential operators. In Proc. Int. Symp. Symbolic Algebraic Comput. (ISSAC'94), pages 336-340. M. Giesbrecht ed., ACM Press, 1994.

[3] F. Chyzak and B. Salvy. Non-commutative elimination in Ore algebras proves multivariate identities. J. Symb. Comput., 26:187-227, 1998.

[4] M. Janet. Les systéms d'équations aux dérivées partielles. Journal de mathématiques, 83:65-123, 1920.

[5] A. Kandri-Rody and V. Weispfenning. Gröbner bases in algebras of solvable type. J. Symb. Comput., 9:1-26, 1990.

[6] E. Kolchin. Differential algebra and algebraic groups. Academic Press., New York, 1973.

[7] Z. Li and F. Schwarz. Rational solutions of Riccati-like partial differential equations. J. Symb. Comput., 31:691-716, 2001.

[8] A. Rosenfeld. Specializations in differential algebra. Trans. Amer. Math. Soc., 90:394-407, 1959.

[9] M. Saito, B. Sturmfels, and N. Takayama. Gröbner deformations of hypergeometric differential equations. Springer-Verlag, Berlin Heidelberg New York, 2000.

[10] L. Schlesinger. Handbuch der Theorie der linearen Differentialgleichungen. Teubner, Leipzig, 1895.

[11] F. Schwarz. A factorization algorithm for linear ordinary differential equations. In Proc. Int. Symp. on Symbolic Algebraic Comput. (ISSAC'89), pages 17-25. G. Gonnet (ed.), ACM Press, 1989.

[12] F. Schwarz. Reduction and completion algorithms for partial differential equations. In Proc. Int. Symp. on Symbolic Algebraic Comput. (ISSAC'92), pages 49-56. P. Wang (ed.), ACM Press, 1992.

[13] F. Schwarz. Janet bases for symmetry groups. In Gröbner bases and applications, London Math. Soc. Lecture Notes Series 251., pages 221-234. B. Buchberger, F. Winkler (eds.), Cambridge University Press, 1998.

[14] S. Tsarev. Factorization of overdetermined systems of linear partial differential equations with finite dimensional solution space. In Proc. 4th Int. Workshop Computer Algebra Scient. Comput. (CASC-2001), pages 529-539. V. Ganzha, E. Mayr, V. Vorozhtsov (eds), Spring-Verlag, 2001. 